\title{
An Analytically Based Approach for Evaluating the Impact of the Noise on the Microwave Imaging Detection
}

\author{
Behnaz Sohani $^{1}$, Adam Degaichia Abdallah ${ }^{1}$, Gianluigi Tiberi ${ }^{1}$, \\ Navid Ghavami ${ }^{2}$, Mohammad Ghavami ${ }^{1}$, and Sandra Dudley ${ }^{1}$ \\ ${ }^{1}$ School of Engineering, London South Bank University, London, UK \\ ${ }^{2}$ UBT - Umbria Bioengineering Technologies, Spin Off of University of Perugia, Perugia, Italy
}

\begin{abstract}
In a realistic scenario, it is inevitable to have noise on the images due to the noise from the system's hardware, which results in producing inaccurate images. This paper presents an investigation on the impact of adding noises into the simulation for an Ultra-Wideband (UWB) Microwave Imaging (MWI) procedure based on the Huygens principle (HP). A comparison between uniform and Gaussian noises at different amplitudes is provided, with the aim of investigating the detection process for applications such as bone fracture detection. This is done using analytical simulations. To construct the electric field at the perimeter of the external cylinder, simulations have been run mimicking UWB signals transmitted onto a simulated cylindrical bonemimicking phantom containing an inclusion with different dielectric properties. This field was simulated using MATLAB and generated a value for the electric field at frequencies between 3 and $5 \mathrm{GHz}$. To investigate the impact of noise on the detection capability, two types of common noises have been applied to the signal at different amplitudes. The resulting images have visually been compared and the imaging performance has also been analysed using an image quantification metric, signal-to-clutter ratio (SCR). The impact of noise on the detection capability was quantified using this image quantification metric.
\end{abstract}

\section{INTRODUCTION}

MWI has attracted considerable interest as a diagnostic tool due to non-ionising radiation and the true potential in offering compact and low-cost imaging devices. This technique has been proposed as a complementary approach to well-established procedures (X-rays, computed tomography (CT), and magnetic resonance imaging (MRI)) for diagnosis and monitoring purposes such as diagnosis of bone conditions, including fractures and joint abnormalities, especially for frequent screening and monitoring [1]. In addition, microwave biomedical imaging can be also applied to other anatomical districts, such as brain stroke detection and breast cancer detection $[2,3]$.

MWI can be used for the detection and monitoring of a variety of diseases since there is contrast at microwave frequencies between the dielectric properties of healthy tissues and tissues with lesions $[4,5]$. Some MWI prototypes have been proposed such as $[1,3]$, where a very simple hardware setup has been implemented, i.e., one transmitting antenna and one receiving antenna -coupled through a vector network analyser (VNA)-, rotate in free space around the object to collect the signals in a multi-bistatic fashion [6]. The MWI procedures rely on algorithms to process the electric field measured at several points around the perimeter of a media.

In a realistic scenario, it is inevitable to have noise on the images due to the noise from the system's hardware and wideband uniform noise and this has resulted in producing inaccurate images (as the noise can even mask the inclusion). To prevent the noise from doing so, it is recommended to either increase the power of the input signal or reduce the level of noise in the system. This study will explore the impact of noise on the detection capability by applying two types of common noises -Gaussian noise and uniform noise- to the signal at different amplitudes. This is done using analytical simulations. To construct the electric field at the perimeter of the external cylinder, simulations have been run mimicking UWB signals transmitted onto a simulated cylindrical bonemimicking phantom containing an inclusion with different dielectric properties. This field was simulated using MATLAB and generated a value for the electric field at frequencies between 3 and $5 \mathrm{GHz}$. To provide a valid comparison between the obtained results of adding these noises, an image quantification metric, signal-to-clutter ratio (SCR) has been introduced and calculated. The quantification of achieved images has been performed by employing this parameter. The impact of different amplitudes of Amplifier noise (Gaussian noise) and Quantisation noise (uniform noise), in 3-5 GHz range, for lesion detection has been investigated. 


\section{MATERIALS AND METHODS}

\subsection{Theoretical Framework}

MWI has previously been extremely studied and researched as a complementary modality for brain stroke detection and breast cancer screening $[2,3]$. Such approaches are mainly on the basis of the reported inherent contrast of dielectric properties of abnormal and healthy tissues over the microwave frequency range. Evidence-based research and methodology has been verified for brain imaging and breast cancer detection $[2,3]$ and [6] which strongly supports our approach for bone lesion detection (considering the same dielectric contrast variation for the bone lesion/fracture).

It has been presented in [3] and [7] that Eq. (1) is able to capture the contrast, i.e., mismatch boundaries, and locate an inclusion within the volume. This research applied a method discussed in [8] which uses Huygens Principle (HP) to forward propagate the waves [9]. The use of HP avoids the obligation of having to solve complex inverse problems, which can significantly increase the computational overhead, elaboration times and possibly cause false solutions.

$$
E_{\mathrm{HP}, 2 \mathrm{D}}^{\mathrm{rcstr}}\left(\rho, \phi ; t x_{m} ; f\right)=\Delta_{s} \sum_{n p=1}^{N_{\mathrm{PT}}} E_{n p, t x_{m}}^{\mathrm{known}} G\left(k_{1}\left|\vec{\rho}_{n p}-\vec{\rho}\right|\right)
$$

In this paper, a simulated waveform is constructed applying the principles introduced by Parrikar et al. [10]. This waveform is transmitted from a line source external to the cylinder and received at a point on the radius, external or at the edge of the cylinder. The electric field $E$ is achieved through Eq. (1) by summing the known Electric Field $E_{n p, t x_{m}}^{\text {known }}$ at $n p$ points on the perimeter. In the above equation, $\vec{\rho} \equiv(\rho, \phi)$ is the observation point; $\Delta_{s}$ is the spatial sampling; $k_{1}$ indicates the wave number of the media constituting the cylinder and $t x_{m}$ represents the transmitting line source operating at frequency $f$.

In Eq. (1), Green's function $G$ is applied to propagate the field. $E_{\mathrm{HP}, 2 \mathrm{D}}^{\mathrm{rcstr}}$ stands for the "reconstructed" internal electric field and has been shown by the string "rcstr", whilst the string HP indicates that Huygens based procedure will be employed in Eq. (1).

If we apply $N_{F}$ frequencies $f_{i}$, the intensity of the final image $I$ can be achieved mathematically through the Eq. (2), i.e., by adding incoherently all the solutions:

$$
I\left(\rho, \phi ; t x_{m}\right)=\frac{1}{B} \sum_{i=1}^{N_{F}} \Delta_{f}\left|E_{\mathrm{HP}, 2 \mathrm{D}}^{\mathrm{rcstr}}\left(\rho, \phi ; t x_{m} ; f_{i}\right)\right|^{2}
$$

where $B$ and $\Delta_{f}$ are the bandwidth and frequency sampling, respectively. It should be mentioned that measurements are taken from multiple transmission sources.

The electric field is calculated applying Eq. (1) to form the matrix $t_{1}$ and the process is repeated for a different position of the transmitter $t_{2}$. In more details, the simulation is set up to replicate a signal from two transmitters, positioned $4.5^{\circ}$ apart on the perimeter of the cylinder. The image is then constructed employing the matrix $d$ which only shows the differences and can be constructed with the following equation:

$$
\mathbf{d}_{i, j}=\mathbf{t}_{1_{i, j}}-\mathbf{t}_{2_{i, j}}
$$

where $t_{1}$ and $t_{2}$ are the results matrices from transmitters one (1) and two (2), respectively.

Next, in this manuscript, the impact of noise on detection performances has been studied. It seems certain that through image acquisition or transmission, numerous factors can be a direct or indirect cause of noise in the image. The noise can undesirably affect the image and result in producing inaccurate images and can even mask the inclusion. To prevent the noise from doing so, it is recommended to either increase the power of the input signal or reduce the level of noise in the system. To investigate the impact of noise on the detection capability, two types of common noises -Amplifier noise (Gaussian noise) and Quantisation noise (uniform noise)- have been applied to the signal at different amplitudes and were quantified using the SCR metric.

\subsection{Image Construction}

Equation (1) is used to generate the field which contains an inclusion in terms of magnitude and phase calculated. The chosen frequency range is between 3 and $5 \mathrm{GHz}$ with a step of $1000 \mathrm{MHz}$. These values of the frequency range and its step will be adjusted to meet the requirements.

Next, to investigate the impact of the noise on the detection performances, we have simulated and considered a multi-layered phantom. Here as an example for bone phantom, which comprises two layers which include: 
I) An external cylinder representing the bone marrow tissue (radius $=55 \mathrm{~mm}$, relative dielectric constant $\varepsilon_{r}=4$ and conductivity $\sigma=1 \mathrm{~S} / \mathrm{m}$ ), and

II) An internal cylindrical inclusion representing the bone fracture/lesion tissue -assumed here as blood- (radius $=0.5 \mathrm{~mm}$, relative dielectric constant $\varepsilon_{r}=60$, conductivity $\sigma=2 \mathrm{~S} / \mathrm{m}$ and located $20 \mathrm{~mm}$ from the centre of the external cylinder).

Two types of image noises that have been used in this study include Amplifier noise (Gaussian noise) and Quantisation noise (uniform noise).

Then, a comparison of different applied noises has been conducted by simulating the waveform of a cylinder with an inclusion. A 'Resulting Image' has been constructed employing the method described above in Eq. (3), without applying the noises. This Resulting Image was then used as a reference image for comparisons. For the simulations, as discussed above, the dielectric constant and conductivity of the inclusion have been assumed to be significantly greater than the surrounding material.

A simulation was run to construct the electric field measured at the perimeter of a cylinder with an inclusion. This field was simulated using MATLAB and generated a value for the electric field at 80 points around the circumference of a cylinder for 1101 discrete frequencies between 3 and 5 GHz. Fig. 1 indicates that the inclusion has been successfully detected.

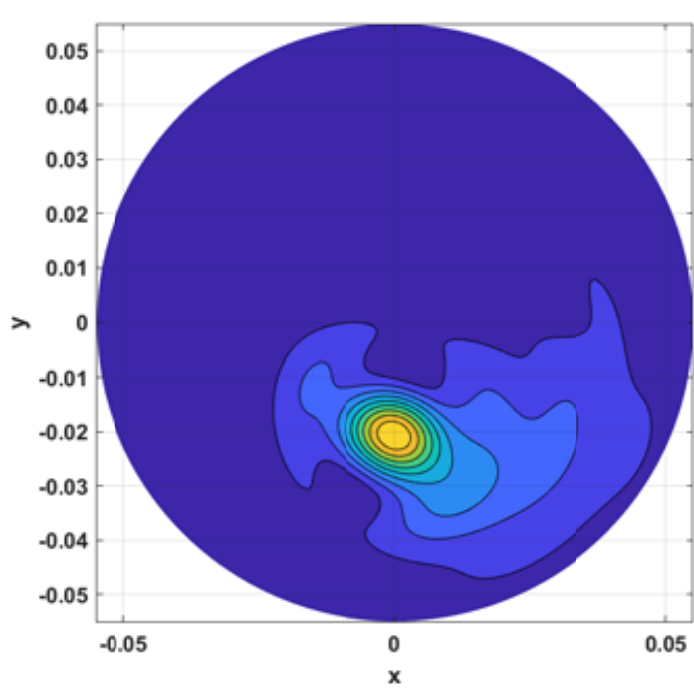

(a)

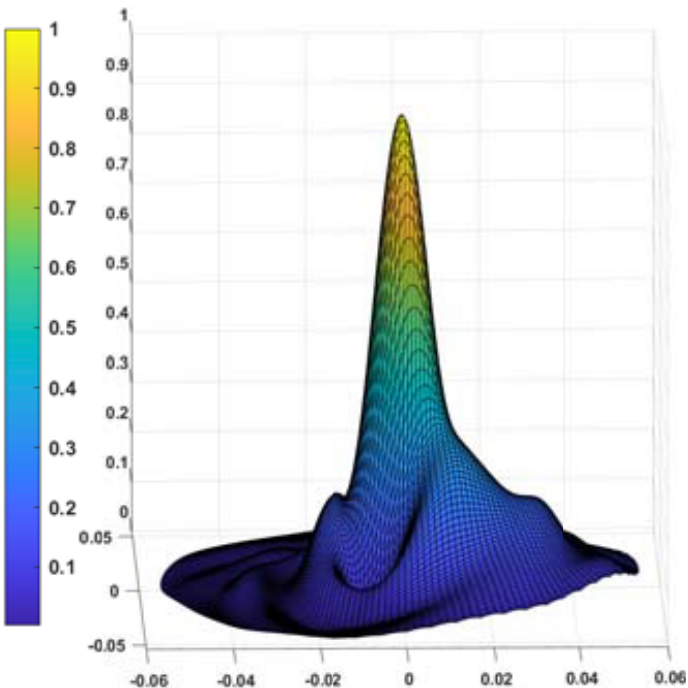

(b)

Figure 1: (a) A noise-free image of a cylinder with an inclusion emulating a bone fracture. (b) Microwave image of a noise-free inclusion. Images are displayed following normalisation to the maximum value and converting intensity values lower than 0.5 to $0 . x$ and $y$ axes are in meters, while the intensity has an arbitrary unit.

It is important to point out that the data here has been adjusted and normalised to produce an output from 0 to 1 , with 0 representing the minimum possible data point and 1 the maximum. This would mean that the image is adjusted by forcing the intensity values below 0.5 to zero. The visualisation and comparison would be relatively easier by using adjusted and normalised data.

The transmitting signal has been set on the perimeter of the circle at $x=-0.07, y=0$. The inclusion can be seen in this image. The detection has been achieved in simulations, after using an imaging algorithm based on HP. Moreover, it proves the functionality of the concept and the possibility of detecting an inclusion for MWI.

\subsection{Amplifier Noise (Gaussian Noise)}

This common sort of noise results from the contributions of many independent signals. This noise model is additive in nature [11] and follows Gaussian distribution. The standard model of amplifier noise is additive, Gaussian, independent of the signal intensity [12]. In most applications, the input signal/the signal to be modelled often contains Gaussian noise. Gaussian noise tends to flatten the signal and distort the correlation function. Therefore, the signal spectrum will come to be flatter than the Original one [13]. The noise, especially in medical images can be modelled using 
mathematical equations which are presented in [11]. Gaussian noise generates an array whose elements are normal with a zero mean and unit variance [14]. The method used in this study is adapted from [15]. By using the MATLAB function awgnor rand, white Gaussian noise to the input signal will be generated [16].

If an input signal $x_{k}$ is contaminated by white Gaussian noise $v_{k}$, the Gaussian noisy signal, which is denoted as $z_{k}$, can be calculated using the following equation:

$$
\mathbf{z}_{k}=\mathbf{x}_{k}+\text { amplitude } \times \operatorname{rand}\left(\mathbf{x}_{k}\right)
$$

It should be highlighted that for the Gaussian noise the function rand will be used.

The code will be executed at different amplitudes of noise. The aim is to investigate the impact of noise under realistic values. The realistic amplitude values of Gaussian noise were adapted from $[15]$. The values are as follows:

- Gaussian noise: at $-40 \mathrm{~dB},-26.02 \mathrm{~dB},-20 \mathrm{~dB},-16.48 \mathrm{~dB}$.

\subsection{Quantisation Noise (Uniform Noise)}

To model measurement noise, uniform noise was added to the squared $E$-field amplitudes on the emulated sensors; the standard deviation of this noise was quantified relative to the peak $E$-field amplitude over the whole measurement plane.

Uniform noise generates an array of uniformly distributed numbers with values between 0 and 1 [14]. By using the MATLAB function rand [16], it will generate a uniform noise to the input signal. A random number will be added to every element of the matrix $d, 10 \%$ of the signal is noisy at every element. An amplitude of the noise compared to the input signal is created which will be varied and investigated.

If an input signal $x_{k}$ is contaminated by uniform noise, the uniform noisy signal, which is denoted as $z_{k}$, can be calculated using the following equation:

$$
\mathbf{z}_{k}=\mathbf{x}_{k}+\text { amplitude } \times \operatorname{rand}\left(\mathbf{x}_{k}\right)
$$

It should be pointed out that for the uniform noise the function rand will be used. For the uniform noise, the values were picked randomly with an increment of 0.1 . The values are as follows:

- Uniform noise: at $-20 \mathrm{~dB},-13.98 \mathrm{~dB},-9.12 \mathrm{~dB},-6.02 \mathrm{~dB}$.

\subsection{Comparison Method}

Imaging performance has been investigated through image quantification. As comparisons are to be drawn between differing images, it is necessary to create a quantifiable measurement system that can be able to be applied to make a comparison between images. It is often problematic to create a useful quantifiable number to measure an image. Whilst humans are good at recognising contrasts and patterns in an image, a machine must be trained for each process, and this uses considerable computing power. For the purposes of this experiment, to provide a valid comparison between the obtained results of adding these noises, an image quantification metric has been introduced and calculated to make the comparison between the results. The metric for detection accuracy is the SCR.

The evaluation of this metric has been performed by calculating Eq. (2) and increasing the amplitudes of noise in different levels, to evaluate to what extent the inclusion can still be detected after applying two types of noises: Amplifier noise (Gaussian noise) and Quantisation noise (uniform noise).

The analysis of SCR with respect to the band of 3 to $5 \mathrm{GHz}$ will be presented.

\subsection{Signal-to-Clutter Ratio (SCR)}

In practice, for evaluating the performance of the imaging, a quantitative metric is used in this research, which is called the SCR. SCR has been defined as the ratio between maximum intensity evaluated in the area of the lesion divided by the maximum intensity outside the area of the lesion. Clutter derives from unfavourable scatter from substances in the radar beam which are not objectives and has been considered by numerous distributions. As the images may be comprised by clutters, it is applicable to apply a quantifiable measurement parameter to compare and quantify the performance in detection.

Usually, the SCR has been referred to as the ratio of the maximum target/objective response to the maximum clutter response. In this study, SCR is introduced and applied as the ratio between 
the maximum intensity evaluated in the area of the lesion divided by the maximum intensity outside the area of the lesion [17]. The SC measures the strength and precision of the results and is extremely useful to use in comparisons.

Table 1: The comparison results of adding gaussian noise and uniform noise at different amplitudes with respect to $\mathrm{SCR}$, with inclusion at $\pi / 2$ radians.

\begin{tabular}{|c|c|c|c|c|}
\hline Noise-free image & \multicolumn{2}{|c|}{ Quantisation noise (uniform noise) } & \multicolumn{2}{c|}{ Amplifier noise (Gaussian noise) } \\
\hline SCR & Amplitude (dB) & SCR & Amplitude (dB) & SCR \\
\hline \multirow{4}{*}{6.65} & -20 & 3.46 & -40 & 3.43 \\
\cline { 2 - 5 } & -13.98 & 3.39 & -26.02 & 3.23 \\
\cline { 2 - 5 } & -9.12 & 1.92 & -20 & 2.50 \\
\cline { 2 - 5 } & -6.02 & 1.46 & -16.48 & 1.11 \\
\hline
\end{tabular}

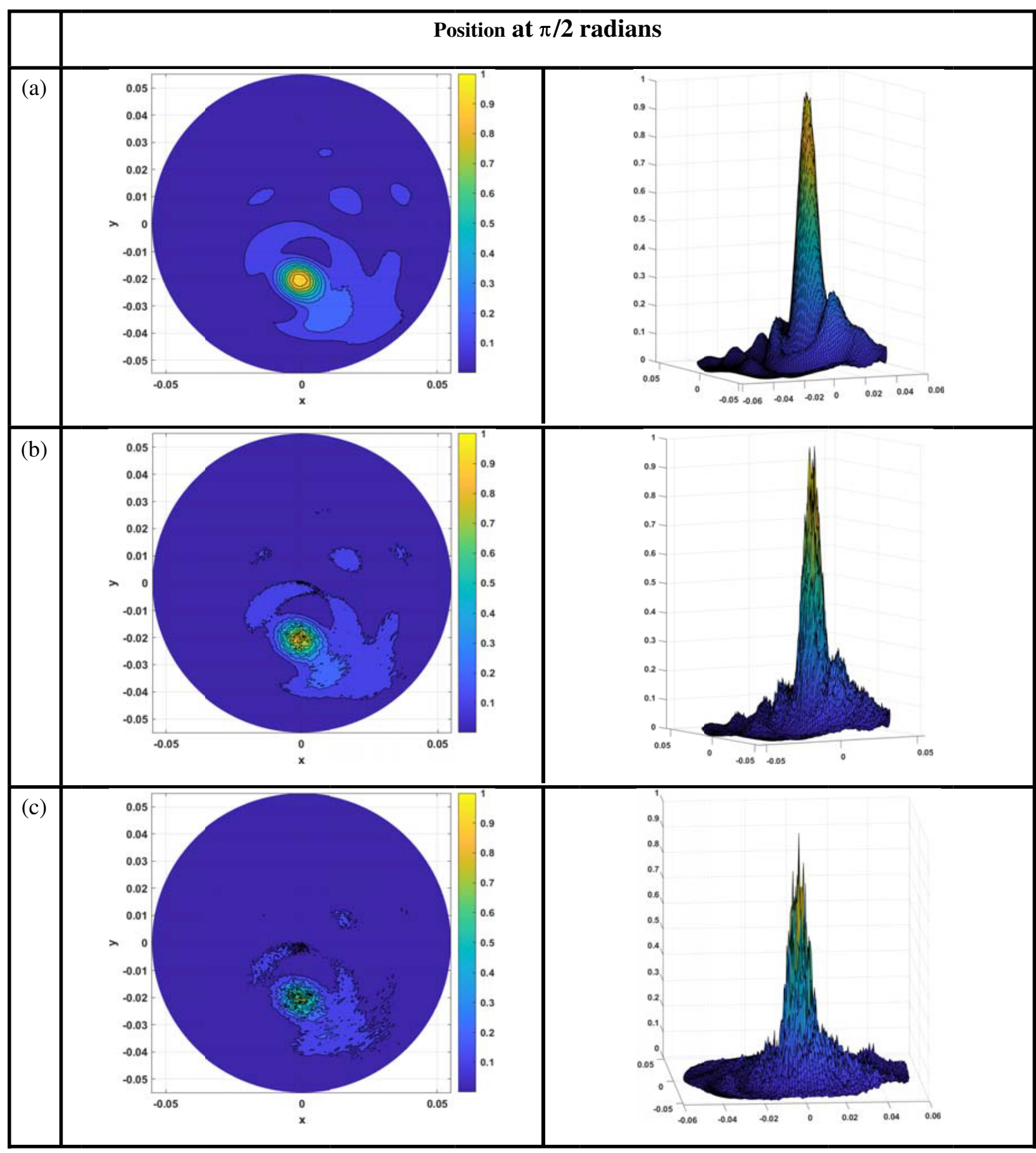




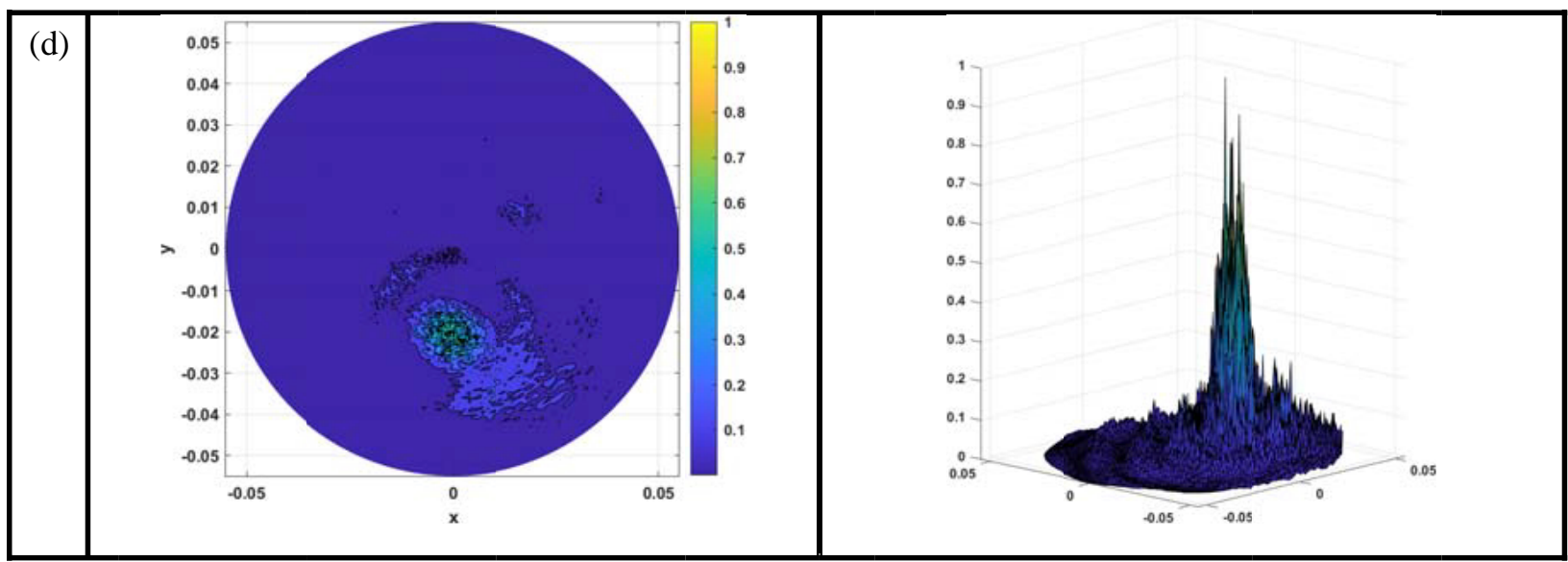

Figure 2: The resulting image for an inclusion emulating a bone fracture at $\pi / 2$ radians after applying amplifier noise (Gaussian noise) at different amplitudes of (a) $-40 \mathrm{~dB}$, (b) $-26.02 \mathrm{~dB}$, (c) $-20 \mathrm{~dB},(\mathrm{~d})-16.48 \mathrm{~dB}$. Images are displayed following normalisation to the maximum value and converting intensity values lower than 0.5 to $0 . x$ and $y$ axes are in meters, while the intensity has an arbitrary unit.

\section{RESULTS}

The system's hardware -such as imaging sensors- may be affected by environmental conditions during image acquisition. It is inevitable to have noise on the images since the wideband communications components contain the uniform noise. Noise is also caused by other natural sources such as atoms vibrations. Noise can decrease the image quality and degrade the image at the time of capturing or transmission of the image, and it may even mask the inclusion. To this end, different

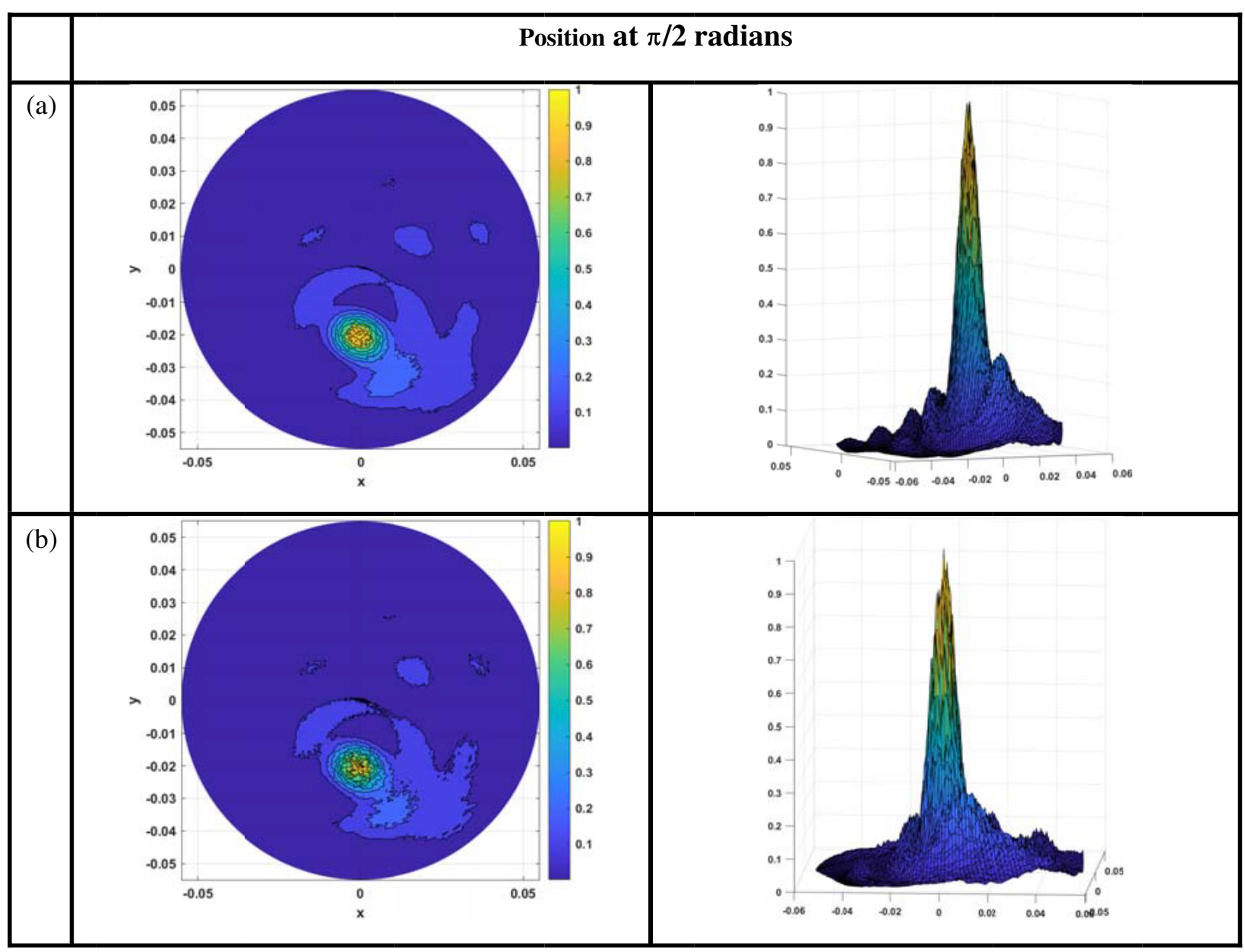




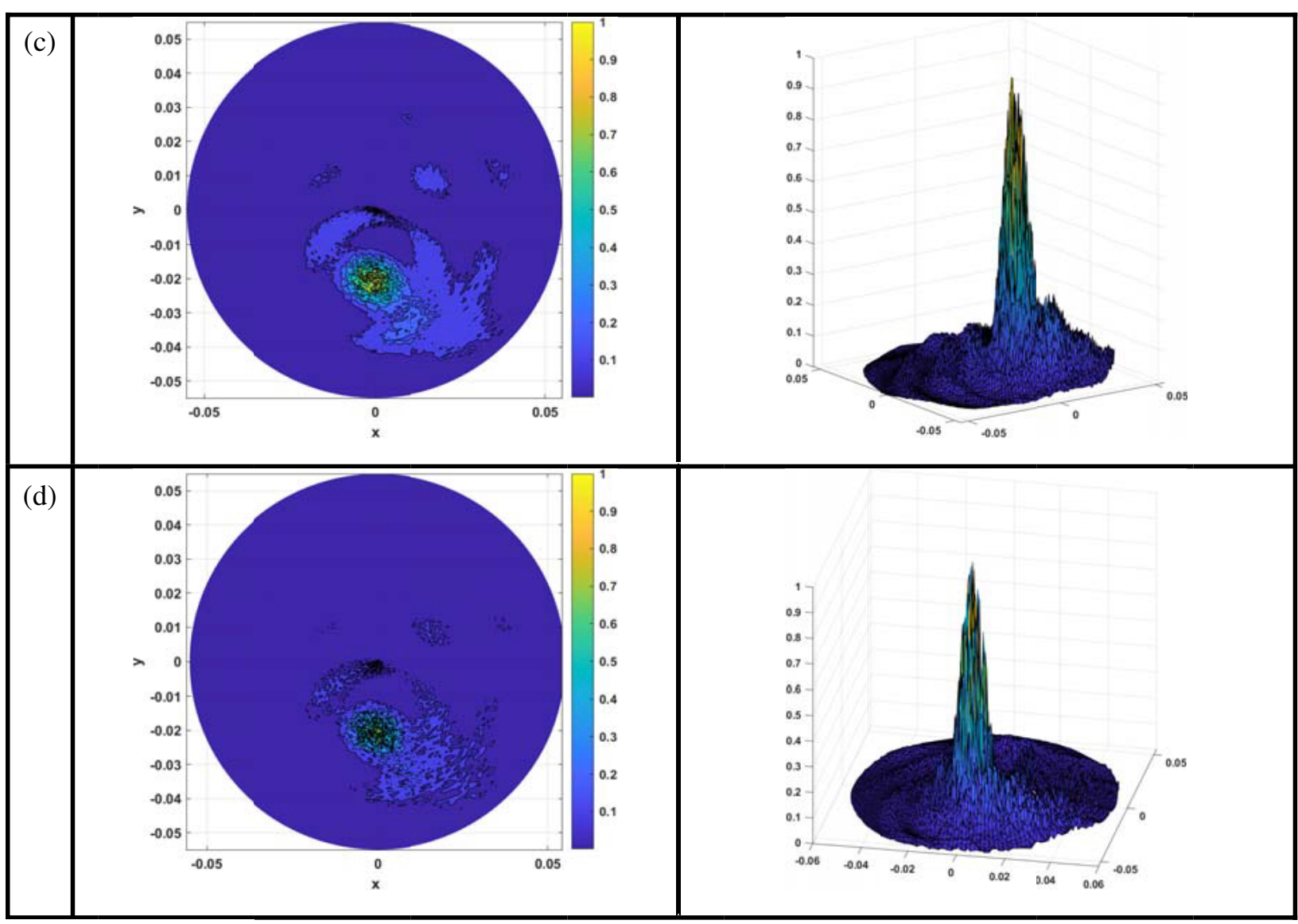

Figure 3: The resulting image for an inclusion emulating a bone fracture at $\pi / 2$ radians after applying Quantisation noise (uniform noise) at different amplitudes of (a) $-20 \mathrm{~dB}$, (b) $-13.98 \mathrm{~dB},(\mathrm{c})-9.12 \mathrm{~dB}$, (d) $-6.02 \mathrm{~dB}$. Images are displayed following normalisation to the maximum value and converting intensity values lower than 0.5 to $0 . x$ and $y$ axes are in meters, while the intensity has an arbitrary unit.

amplitudes of noise were used to see to what extent the inclusion can still be detected. In this paper, our aim is to study the impact of adding noises into the simulation for a MWI procedure based on the HP and prove that our proposed method has the potential to successfully detect the inclusion (here the inclusion is mimicking the bone lesion).

A comparison of different applied noises was conducted by simulating the waveform of a cylinder with an inclusion mimicking bone lesion tissue. The simulation was run by applying each of two noises. The results are presented for inclusion at $\pi / 2$ radians (Table 1$)$. The signal-to-clutter ratio (SCR) metric is expressed in abbreviated form and presented in decibels $(\mathrm{dB})$.

Figure 2 represents the resulting images for an inclusion emulating a bone fracture at $\pi / 2$ radians after applying Amplifier noise (Gaussian noise) at different amplitudes of (a) $-40 \mathrm{~dB}$, (b) $-26.02 \mathrm{~dB}$, (c) $-20 \mathrm{~dB},(\mathrm{~d})-16.48 \mathrm{~dB}$. Figure 3 indicates the images after applying Quantisation noise (uniform noise) at different amplitudes of (a) $-20 \mathrm{~dB},(\mathrm{~b})-13.98 \mathrm{~dB},(\mathrm{c})-9.12 \mathrm{~dB},(\mathrm{~d})-6.02 \mathrm{~dB}$.

It is worth noting that, the images presented in this manuscript were normalised and adjusted, forcing the intensity values below 0.5 to zero, whilst SCR was always calculated before performing the image adjustment.

\section{DISCUSSION AND CONCLUSION}

The detection capabilities can be affected by problems such as the build-up of noise and signal distortion during the processing of images. This study intended to compare two different applied noises, Amplifier noise (Gaussian noise) and Quantisation noise (uniform noise) to assess to what extent the inclusion can still be detected after applying these two types of noises.

The inclusion can be detected after applying Amplifier noise (Gaussian noise) at different amplitudes. However, when the amplitude is increased, more spikes appear and the inclusion is barely detected. Thus, any Gaussian noise level higher than 10\% of the input signal can significantly affect 
the detection capabilities and highly degrade the image. It should be highlighted that the Amplifier noise (Gaussian noise) has stronger impacts on the detection capabilities than the Quantisation noise (uniform noise). This can be concluded by comparing Fig. 2. and Fig. 3. The maximum Gaussian noise level on which the inclusion can be detected is $10 \%$, whilst for the uniform noise, the maximum amplitude level is approximately $35 \%$.

The results show that the highest SCR achieved is $6.65 \mathrm{~dB}$ belonging to the noise-free image evidently. It is verified that after applying Amplifier noise (Gaussian noise) and Quantisation noise (uniform noise), the highest SCR which was attained for the Gaussian noise and uniform noise was $3.43 \mathrm{~dB}$ (at the amplitude of $-40 \mathrm{~dB}$ ) and $3.46 \mathrm{~dB}$ (at the amplitude of $-20 \mathrm{~dB}$ ), respectively. These two were acquired at the lowest level of amplitude. The more the amplitude was increased the lower the SCR was. The detection capability is unattainable if the SCR is below $2.50 \mathrm{~dB}$ (at the amplitude of $-20 \mathrm{~dB}$ ) and $1.92 \mathrm{~dB}$ (at the amplitude of $-9.12 \mathrm{~dB}$ ) for Gaussian noise and uniform noise, respectively. This would mean that the power has been reduced. As mentioned in [18], a 3 - $\mathrm{dB}$ loss corresponds to halving the power. This also means that the signal lost half of its power due to the noise. Also, with decreasing the SCR, the system detectability will be substantially reduced and artefacts will be increased in the images.

To summarise, the SCR will decrease by increasing the amplitude. Thus, to improve the data acquisition, we must either increase the power of the input signal or reduce the noise level from the system. This can be achieved by adding noise filters into the system and this will be discussed in more details in the future recommendation.

\section{REFERENCES}

1. Nikolova, N. K., "Microwave imaging for breast cancer," IEEE Microw. Mag., Vol. 12, No. 7, 2011, doi: 10.1109/MMM.2011.942702.

2. Sohani, B., et al., "Developing artefact removal algorithms to process data from a microwave imaging device for haemorrhagic stroke detection," Sensors (Switzerland), Vol. 20, No. 19, 2020, doi: $10.3390 / \mathrm{s} 20195545$.

3. Sani, L., et al., "Novel microwave apparatus for breast lesions detection: Preliminary clinical results," Biomed. Signal Process. Control, Vol. 52, 2019, doi: 10.1016/j.bspc.2019.04.025.

4. Semenov, S., "Microwave tomography: Review of the progress towards clinical applications," Philosophical Transactions of the Royal Society A: Mathematical, Physical and Engineering Sciences, Vol. 367, No. 1900. 2009, doi: 10.1098/rsta.2009.0092.

5. Lazebnik, M., et al., "A large-scale study of the ultrawideband microwave dielectric properties of normal, benign and malignant breast tissues obtained from cancer surgeries," Phys. Med. Biol., Vol. 52, No. 20, 2007, doi: 10.1088/0031-9155/52/20/002.

6. Sohani, B., et al., "Detection of haemorrhagic stroke in simulation and realistic 3-D human head phantom using microwave imaging," Biomed. Signal Process. Control, Vol. 61, 2020, doi: 10.1016/j.bspc.2020.102001.

7. Ghavami, N., P. P. Smith, G. Tiberi, D. Edwards, and I. Craddock, "Non-iterative beamforming based on Huygens principle for multistatic ultrawide band radar: Application to breast imaging," IET Microwaves, Antennas Propag., Vol. 9, No. 12, 2015, doi: 10.1049/ietmap.2014.0621.

8. Ghavami, N., G. Tiberi, D. J. Edwards, and A. Monorchio, "UWB microwave imaging of objects with canonical shape," IEEE Trans. Antennas Propag., Vol. 60, No. 1, 2012, doi: 10.1109/TAP.2011.2167905.

9. Enders, P., "Huygens' principle as universal model of propagation," Lat. Am. J. Phys. Educ., Vol. 3, No. 1, 2009.

10. Parrikar, R. P., A. A. Kishk, and A. Z. Elsherbeni, "Scattering from an impedance cylinder embedded in a nonconcentric dielectric cylinder," IEE Proc. H, Microwaves, Antennas Propag., Vol. 138, No. 2, 1991, doi: 10.1049/ip-h-2.1991.0028.

11. Patidar, P., M. Gupta, S. Srivastava, and A. K. Nagawat, "Image de-noising by various filters for different noise," Int. J. Comput. Appl., Vol. 9, No. 4, 2010, doi: 10.5120/1370-1846.

12. Boncelet, C., "Image noise models," Handbook of Image and Video Processing, 2005.

13. Gravel, P., G. Beaudoin, and J. A. De Guise, "A method for modeling noise in medical images," IEEE Trans. Med. Imaging, Vol. 23, No. 10, 2004, doi: 10.1109/TMI.2004.832656.

14. Feng, D. and M. Q. Feng, "Appendix: Fundamentals of digital image processing using MATLAB," Computer Vision for Structural Dynamics and Health Monitoring, 2020. 
15. Kociołek, M., M. Strzelecki, and R. Obuchowicz, "Does image normalization and intensity resolution impact texture classification?," Comput. Med. Imaging Graph., Vol. 81, 2020, doi: 10.1016/j.compmedimag.2020.101716.

16. MATLAB Documentation Centre, "Add white Gaussian noise to signal - MATLAB awgnMathWorks United Kingdom," https://uk.mathworks.com/help/comm/ref/awgn.html (accessed Mar. 5, 2021).

17. Fear, E. C., X. Li, S. C. Hagness, and M. A. Stuchly, "Confocal microwave imaging for breast cancer detection: Localization of tumors in three dimensions," IEEE Trans. Biomed. Eng., Vol. 49, No. 8, 2002, doi: 10.1109/TBME.2002.800759.

18. Anttalainen, T., Introduction to Communication Networks, Artech House, 2014. 